\title{
Editorial
}

\section{Signal Processing with High Complexity: Prototyping and Industrial Design}

\author{
Markus Rupp, ${ }^{1}$ Thomas Kaiser, ${ }^{2}$ Jean-Francois Nezan, ${ }^{3}$ and Gerhard Schmidt ${ }^{4}$ \\ ${ }^{1}$ Institute for Communication and RF Engineering, Vienna University of Technology, Gusshausstrasse 25/389, 1040 Vienna, Austria \\ ${ }^{2}$ Institut für Kommunikationstechnik, Leibniz Universität Hannover, Appelstrasse 9a, 30167 Hannover, Germany \\ ${ }^{3}$ IETR/Image Group Lab, France \\ ${ }^{4}$ Harman/Becker Automotive Systems, 89077 Ulm, Germany
}

Received 10 July 2006; Accepted 11 July 2006

Copyright (c) 2006 Markus Rupp et al. This is an open access article distributed under the Creative Commons Attribution License, which permits unrestricted use, distribution, and reproduction in any medium, provided the original work is properly cited.

Some modern applications require an extraordinary large amount of complexity in signal processing algorithms. For example, the 3 rd generation of wireless cellular systems is expected to require 1000 times more complexity when compared to its 2nd generation predecessors, and future $3 \mathrm{GPP}$ standards will aim for even more number-crunching applications. Video and multimedia applications do not only drive the complexity to new peaks in wired and wireless systems but also in personal and home devices. Also in acoustics, modern hearing aids, or algorithms for dereverberation of rooms, blind source separation and multichannel echo cancellation are complexity hungry. At the same time the anticipated products also put on additional constraints like size and power consumption when mobile and thus battery powered. Furthermore, due to new developments in electro-acoustic transducer design, it is possible to design very small and effective loudspeakers. Unfortunately, the linearity assumption does not hold any more for this kind of loudspeakers, leading to computationally demanding nonlinear cancellation and equalization algorithms.

Since standard design techniques would either consume too much time or not result in solutions satisfying all constraints, more efficient development techniques are required to speed up this crucial phase. In general such developments are rather expensive due to the required extraordinary high complexity. Thus, de-risking of a future product based on rapid prototyping is often an alternative approach. However, since prototyping would delay the development, it often makes only sense when it is well embedded in the product design process. Rapid prototyping has thus evolved by applying new design techniques more suitable to support a quick time to market requirement.
This special issue focuses on new development methods for applications with high complexity in signal processing and on showing the improved design obtained by such methods. Examples of such methods are virtual prototyping, HW/SW partitioning, automatic design flows, float to fix conversions, and automatic testing and verification.

We received seven submissions of which only four were accepted.

In Rapid industrial prototyping and SoC design of $3 G / 4 G$ wireless systems using an HLS methodology the authors Yuanbin Guo et al. present their industrial rapid prototyping experiences on $3 \mathrm{G} / 4 \mathrm{G}$ wireless systems using advanced signal processing algorithms in MIMO-CDMA and MIMO-OFDM systems. Advanced receiver algorithms suitable for implementation are proposed for synchronization, MIMO equalization, and detection, VLSI-oriented complexity reduction is presented. This design experience demonstrates that it is possible to enable an extensive architectural analysis in a short time frame using HLS methodology by abstracting the hardware design iterations to an algorithmic $\mathrm{C} / \mathrm{C}++$ fixedpoint design, which in turn significantly shortens the time to market for wireless systems.

In Generation of embedded hardware/software from system $C$ the authors Salim Ouadjaout and Dominique Houzet present a design flow to reduce the SoC design cost. This design flow unifies hardware and software using a single high level language and thus decreases the manual errors by rewriting design code. It integrates hardware/software (HW/SW) generation tools and an automatic interface synthesis through a custom library of adapters. The approach is validated on a hardware producer/consumer case study and on the design of a given software-radio communication application. 
In Efficient design methods for embedded communication systems the authors Martin Holzer et al. analyze a complete design process to exhibit inefficiencies. The lack of an integrated design methodology is argued. High level characterisation, virtual prototyping, automated hardware/software partitioning, and floating-point to fixed-point data conversion are bottlenecks to solve in such a methodology. For each point, authors present and compare several tools and algorithms leading to an efficient fast prototyping framework. Examples are given in the field of high-complexity communication systems but can be extended to other complex application fields.

In Fixed-point configurable hardware components the authors Romuald Rocher et al. propose a flexible scheme for fixed-point optimization in order to better exploit advances in VLSI technology. After determining the dynamic range and the binary point, a data word-length optimization follows by introducing a suitable user-defined cost function. This central cost function, which, for example, depends on chip area and/or energy consumption, is to be minimized under the constraint of a pre-defined thresholded signal-toquantization noise ratio (SQNR). Through use of analytical models the design time can be significantly reduced. A 128 tap LMS filter design exemplarily explores the fixed-point search space and demonstrates the benefits of the proposed scheme.

\section{Markus Rupp \\ Thomas Kaiser \\ Jean-Francois Nezan \\ Gerhard Schmidt}

\begin{abstract}
Markus Rupp received his Dipl.-Ing. degree in 1988 at the University of Saarbruecken, Germany, and his Dr.-Ing. degree in 1993 at the Technische Universitaet Darmstadt, Germany. He is presently a Full Professor for digital signal processing in mobile communications at the Technical University of Vienna. He is Associate Editor of IEEE Transactions on Signal Processing, EURASIP Journal of Applied Signal Processing, EURASIP Journal on Embedded Systems and is elected AdCom Member of EURASIP. He authored and co-authored more than 200 papers and patents on adaptive filtering, wireless communications, and rapid prototyping.

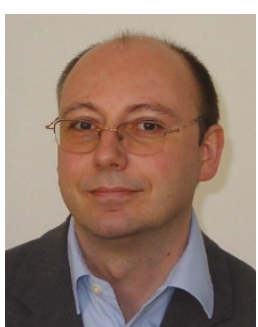

Thomas Kaiser received the Ph.D. degree in 1995 with distinction and the German habilitation degree in 2000, both in electrical engineering from Gerhard-MercatorUniversity Duisburg. In the summer of 2005 he joined Stanford's Smart Antenna Research Group (SARG) as a Visiting Professor. Now he holds a chair on communication systems at the University of Hannover, Germany, and is a founder of the spin-off company mimoOn $\mathrm{GmbH}$. He has published more than 100 papers and has co-edited four books on ultra-wideband and smart

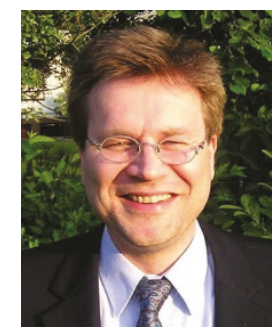

antenna systems. He is the founding Editor-in-Chief of the IEEE Signal Processing Society e-letter. His research interest focuses on applied signal processing with emphasis on multi-antenna systems, especially its applicability to ultra-wideband systems.

Jean-Francois Nezan is an Assistant Professor at National Institute of Applied Sciences of Rennes (INSA) and a member of the IETR laboratory in Rennes. He received his postgraduate certificate in signal, telecommunications, images, and radar sciences from Rennes University in 1999, and his engineering degree in electronic and computer engineering from INSA-Rennes Scientific and Technical University in 1999.

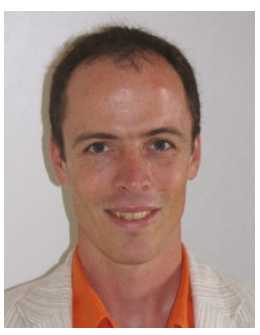
He received his Ph.D. degree in electronics in 2002 from the INSA. His main research interests include image compression algorithms and multi-DSP rapid prototyping.

Gerhard Schmidt received his Dipl.-Ing. degree in 1996 and his Dr.-Ing. degree in 2001, both at Darmstadt University of Technology, Germany. Presently, he is working as a senior research engineer in the acoustic signal processing group at Harman/Becker Automotive Systems in Ulm, Germany. His main research interests include adaptive methods for speech and audio processing.

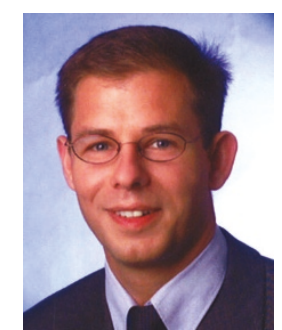

